\title{
Reasons of Vaccine Hesitancy among Pakistanis with Available COVID-19 Vaccine: The Next Challenge in the Fight against COVID-19
}

\section{Wajiha Rizwan1, Muhammad Nasir Rana1, Mulazim Hussain Bukhari' ${ }^{2}$, Asma Mushtaq1, Masood Sadiq ${ }^{1}$}

${ }^{1}$ The Children Hospital/Institute of Child Health, Lahore, Pakistan; ${ }^{2}$ Azad Jammu Kashmir Medical College, Muzaffarabad, Pakistan

Correspondence to: Wajiha Rizwan, drwajiharizwan@gmail.com

Keywords: COVID-19, Vaccine Hesitancy, Vaccine Side Effects, Vaccine and Propaganda, Herd Immunity

Received: May 1, $2021 \quad$ Accepted: June 12, $2021 \quad$ Published: June 15, 2021

Copyright $\odot 2021$ by author(s) and Scientific Research Publishing Inc.

This work is licensed under the Creative Commons Attribution International License (CC BY 4.0).

http://creativecommons.org/licenses/by/4.0/

\section{(c) (i) Open Access}

\section{ABSTRACT}

The government of Pakistan started vaccination campaign for the frontline healthcare providers, as they are more likely to be exposed to COVID-19 patients. In the next step, it extended the free vaccination campaign to include people above 60 and 50 years of age respectively. The drive is now open to all citizens $>18$ years of age. There is a significant reluctance to get vaccinated even though the government has provided it free of cost. The project uses self-reporting method to assess reasons of vaccine hesitancy among Pakistanis and possible solutions to overcome this problem. Concern for serious side effects was the most common issue 154 (48.42\%) reported among all groups of people, including the healthcare workers. The major sources of COVID-19 related information were television 117 (36.8\%) and healthcare professionals 105 (33\%). The participants reported that they can be convinced to get vaccinated if more published data related to vaccine efficacy and safety is available $118(37.1 \%)$, they observe no side effect among vaccine recipients $90(28.3 \%)$ or higher government officials get the same vaccine 39 (12.3\%). Vigorous analysis and publication of data generated is paramount to increase the percentage of people being vaccinated. The healthcare professionals should share evidence-based knowledge related to vaccine efficacy and safety through television and social media to increase acceptance rates and hence, decrease mortality and morbidity from COVID-19 in Pakistan. 


\section{INTRODUCTION}

Pakistan is facing the third wave of COVID-19 pandemic since March 2021. If the vaccination rates remain low, there is a high likelihood of resurgence thus impacting public health and in the long run businesses and revenue of the country. Pakistan currently ranks $120^{\text {th }}$ out of 123 vaccinated countries and lags far behind in vaccination schedules of its community compared to some other Asian and African countries. Vaccine hesitancy remains a barrier to full population inoculation against highly infectious diseases. It may take years to create herd immunity which can be an alarming situation for a country with a population of $\sim 220$ million. Corresponding to the rapid development of COVID-19 vaccines globally concerns about the safety of a rapidly developed vaccine without full clinical trials could be a factor that contributes heavily to the vaccine hesitancy.

Pakistan started vaccination for all public sector front-line healthcare workers across the country, with the Chinese developed Sinopharm vaccine, which is, inactivated viral vaccine. People without a scientific background can easily misinterpret scientific data. Pakistan is quite vulnerable to conspiracy narratives and theories due to lack of access to quality education and easy availability of misinformation to people that is more readily accepted. Pakistan has experienced failures of polio vaccination program because of such claims [1-3]. The propensity for COVID-19 disease spread is high in Pakistan because of a weak healthcare system, high population density, and poor compliance with hygiene practices. There is also concern regarding its safety, side effect profile and adverse reactions stemming from the use of vaccine. It is to be mentioned that till date there has not been a single case of serious side effects reported from the COVID-19 vaccine in Pakistan. People generally believe that they will become infertile; their DNA will be altered, stating that COVID-19 is a grand illusion by the Western countries and a conspiracy against Muslim countries [1, 3-5].

Efficacy data alone is not sufficient enough to understand the skepticism regarding the vaccine. The overall level of trust in the health service providers is critical. People are concerned about getting enrolled in what they perceive a blind study clinical trial, with no one knowing if they will actually get the vaccine or the placebo $[1,2]$.

It is the duty of the government, expert health professionals, social media and news agencies to tell the people about the safety of the vaccine. COVID-19 vaccine development was fast, but it did not skip steps or compromise on safety or efficacy. The clinical trials for the first two COVID-19 vaccines included different races such as Black (about $10 \%$ of participants) and Hispanic (about 20\% of participants), older age groups (about 25\%), and people with conditions such as obesity, diabetes, and heart and respiratory conditions [6]. In addition to hesitancy around the vaccine itself, there is also a sense of indifference among the general public regarding the virus, given Pakistan's relatively low number of deaths and cases of severe disease from COVID-19 $[3,4,6]$.

The side effects of the COVID-19 vaccine are mostly mild and self-resolving. After the vaccination shot, one can experience side effects for e.g., a sore arm, low grade fever or body aches, but cannot catch COVID-19 infection due to vaccine [7]. These symptoms, if they happen at all, usually last only a day or two. They signal the natural response of the body's immune system learning to recognize and fight the SARS-CoV-2 virus. It is observed that there is confusion regarding getting vaccine among lactating or pregnant women and previously COVID-19 infected people. Vaccine is safe for breastfeeding mothers, people who are with weak immune system, even in pregnancies. Pregnant women need to discuss with their doctors before getting COVID-19 vaccine as yet the vaccines have not been tested in pregnant women $[1,2,6-8]$. Current guidelines suggest that people previously infected with COVID-19 still need to be vaccinated as there are chances of reinfection and it is also observed that people who have been vaccinated after having COVID-19 infection have observed a strong immune reaction after the first of the two shots with the Pfizer and Moderna vaccines [7-13]. Vaccine hesitancy is a major challenge to bring an end to this pandemic in countries like Pakistan. Hence, we planned to conduct a survey to find out major concerns of people hesitant to get vaccinated despite free provision of COVID-19 vaccine and to find out possible solution to overcome this problem. 


\section{MATERIALS AND METHODS}

The survey was conducted from $28^{\text {th }}$ March to $5^{\text {th }}$ April 2021. A questionnaire was designed and circulated on social forum (WhatsApp and Facebook) through Google survey system. The participants were above 18 years of age and divided into different age groups (A, B, C, D and E) (Table 1). The participants were informed about voluntary participation with no money paid on participation. Consent was implied through completion of the questionnaire. The inclusion criteria were that the respondents were Pakistani citizens (who were hesitant to get Sinopharm vaccine despite being freely provided to them by the government of Pakistan).

It was a simple cross-sectional survey, consisting of questions that assessed; age, gender, COVID-19 infection status, major reason of vaccine reluctance, knowledge about COVID-19 and COVID-19 vaccination, intention to receive a COVID-19 vaccine on availability, major source of COVID-19 related information and how they could be convinced to get the available vaccine. The participants were also queried about their existing chronic diseases. The categorical variables were expressed as numbers with percentages. The statistical analyses were performed using IBM SPSS Statistics Version 25.

\section{RESULTS}

There were 318 participants who responded in one month, among them 155 (48.7\%) were females and $162(51.3 \%)$ were males. Female to male ratio was $1: 0.8$. There were 5 age groups A, B, C, D and E and the response from each group is shown in Table 1. Professionally the participants mainly comprised of doctors, medical students, other healthcare providers and hospital support staff (Figure 1).

The main source of information were television 117 (36.8\%), health professionals 105 (33\%), Facebook $52(16.3 \%)$ and the government official website 34 (10.6\%) (Figure 2).

The main reason of hesitancy was the fear of known or unknown long-term side effects ( $\mathrm{n}=$ 154-48.42\%). The other important concerns included doubts over vaccine efficacy $(\mathrm{n}=53-16.66 \%)$ and past COVID-19 infection being considered protective against reinfection $(\mathrm{n}=30-9.43 \%)$. It was also observed that participants of COVID-19 vaccine interventional trial $(n=14-4.4 \%)$ had confusion in getting vaccine shot (Table 2). The participants reported that they can be convinced to get Sinopharm vaccine if more published data related to vaccine efficacy and safety is available ( $\mathrm{n}=118-37.1 \%)$, they observe no serious side effect among vaccine recipients $(n=90-28.3 \%)$ or higher government officials get the same vaccine (39-12.3\%) (Table 3 ).

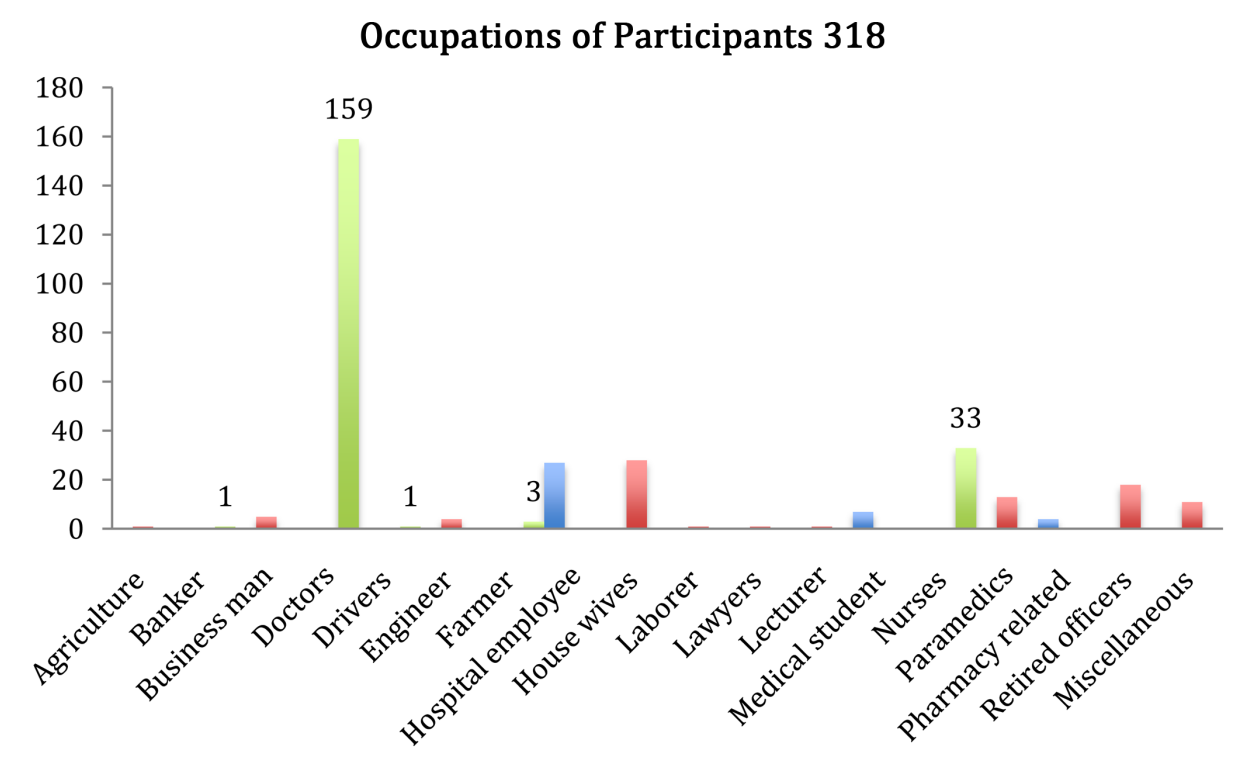

Figure 1. Occupations of participants 318. 


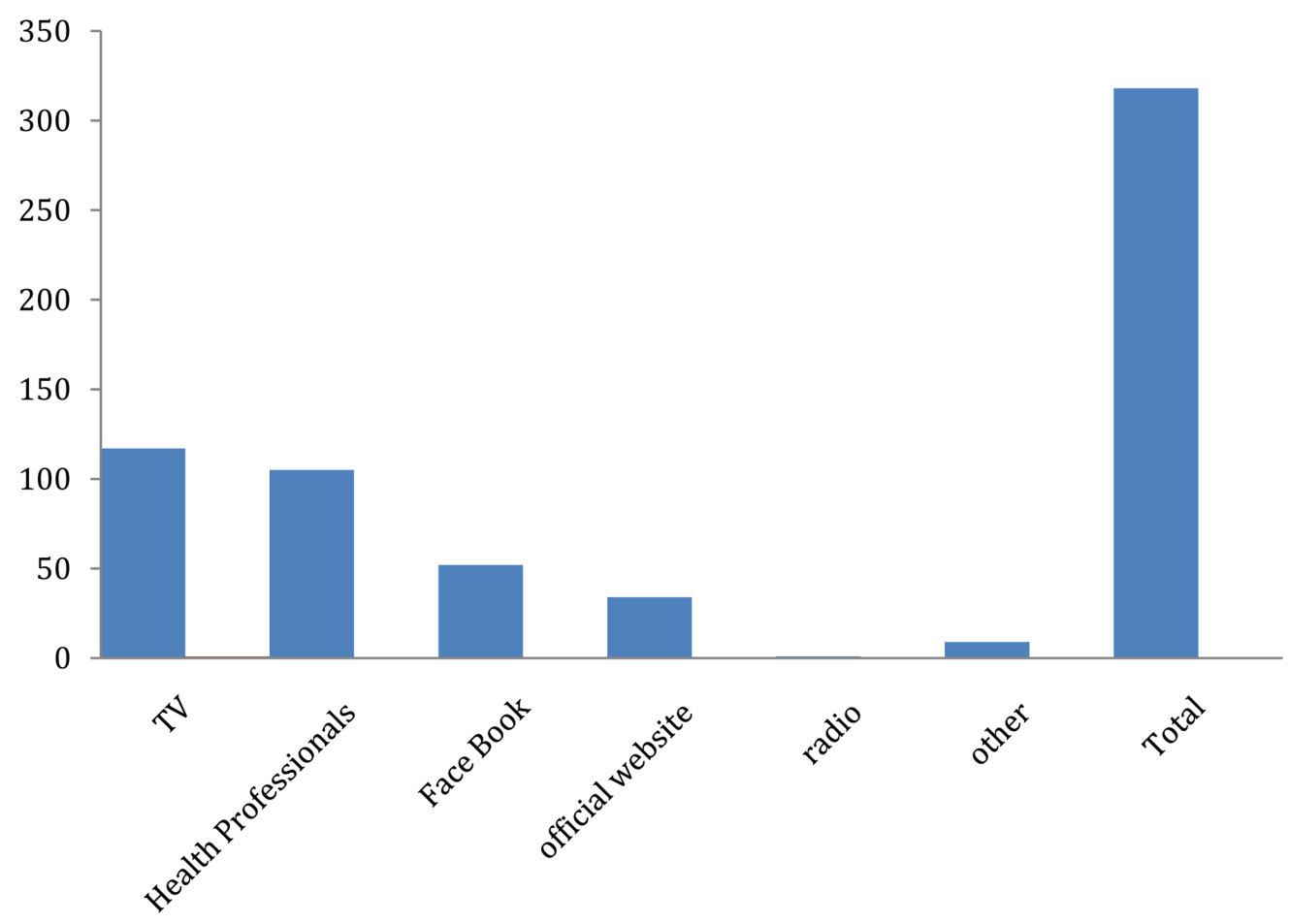

Figure 2. Source of COVID-19 related awareness of the participants.

Table 1. Distribution of the age groups of the participants 318 .

\begin{tabular}{ccc}
\hline Groups & Age & Percentage \\
\hline A & $18-25$ & 24.4 \\
B & $26-35$ & 41.1 \\
C & $36-45$ & 12.3 \\
D & $46-60$ & 12.7 \\
E & $>60$ & 9.5 \\
\hline
\end{tabular}

\section{DISCUSSION}

The success of vaccination program mainly depends on its acceptance among general public and especially the healthcare professionals. Therefore, it is of crucial importance to understand reasons behind their reluctance and to address them by formulating effective strategies. This study is the first ever survey to analyze the causes of vaccine hesitancy in Pakistani population towards COVID-19 vaccination. A sufficient number of healthcare professionals along with general public are hesitant to be vaccinated. The major factors to vaccine hesitancy in our study population include concerns about serious vaccine reactions and side effects and doubts on vaccine efficacy. The findings are consistent with data from other countries reporting similar reasons of hesitancy [12-14].

Television, healthcare professionals and social media is playing an important role in creating awareness about COVID-19 and its preventive strategies including vaccination. Media needs to avoid any exaggerated or amplified statements triggering negative perceptions related to COVID-19 among the general community. Only expert health professionals should be engaged to address queries of the public. Most of participants had reluctance due to lack of published data on efficacy and safety of vaccine as phase III data 
Table 2. Hesitancy with COVID-19 vaccine and response of the participants 318.

\begin{tabular}{|c|c|c|}
\hline Reason of vaccine Hesitancy & Participants number (n) & Percentage (\%) \\
\hline Fear of side effects & 154 & 48.42 \\
\hline Doubt on vaccine efficiency & 53 & 16.66 \\
\hline Already infected with COVID-19 & 30 & 9.43 \\
\hline Distrust on government & 27 & 8.5 \\
\hline Consider Covid-19 and vaccine propaganda & 20 & 6.2 \\
\hline Previously taken part in vaccine trials & 14 & 4.4 \\
\hline Don't know about vaccination & 07 & 2.4 \\
\hline Laziness in visiting vaccination center & 03 & 0.9 \\
\hline Dissatisfaction over quality of vaccine & 02 & 0.6 \\
\hline Afraid due to co morbidities & 02 & 0.6 \\
\hline Doubtful as people even getting positive after vaccination & 02 & 0.6 \\
\hline Lactating mothers & 02 & 0.6 \\
\hline Others & 02 & 0.6 \\
\hline Total & 318 & 100 \\
\hline
\end{tabular}

Table 3. How can participants be convinced to get COVID-19 vaccine.

\begin{tabular}{lcc}
\hline \multicolumn{1}{c}{ You will be convinced to get vaccine if? } & Number of participants & Percentage \\
\hline More published results on vaccine safety and efficacy & 118 & 37.1 \\
I observe no serious side effects in vaccine recipients & 90 & 28.3 \\
Higher government officials take same vaccine & 39 & 12.3 \\
Private good quality vaccine available & 34 & 10.7 \\
Politicians take same vaccine & 14 & 4.4 \\
If compulsory by hospital administration & 5 & 1.6 \\
I don't need as I am immune due to previous infection & 3 & 0.9 \\
I will not get vaccinated as COVID-19 is propaganda & 3 & 0.9 \\
Miscellaneous & 12 & 3.8 \\
\hline
\end{tabular}

of Sinopharm has only been published very recently, after the study had been conducted by Al Kaabi N et al. [15]. The third important factor to avoid vaccination was misconception that previous COVID-19 infection is protective against reinfection and hence they do not require vaccine. The COVID-19 reinfection 
cases are being increasingly reported hence everyone should get vaccinated irrespective of previous COVID-19 infection [16]. We need to say something about fourth reason too. The Government must ensure the importance of vaccination and restore the trust on safe usage of COVID-19 Vaccine to control the current pandemic.

Conspiracy theory was the fifth most crucial factor; with people thinking the vaccine and COVID-19 as just propaganda. Very few ladies considered of pregnancy and lactation, making them non-eligible for vaccine. This might be due to the reason that initially vaccination was refused to this group as no vaccine trial included pregnant and lactating females, hence it is required to encourage pregnant and lactating females to get vaccinated otherwise we will never be able to reach evidence based recommendations [17].

Our study not only points out major causes of hesitancy related to COVID-19 vaccine but also gives insight to possible solutions. Majority believes that if more published data is available on safety and efficacy of COVID-19 vaccine or they observe that recipients had no serious side effects, they can be convinced to receive vaccine shot. Pregnant and lactating women are also waiting for clear instructions from government. Hence worldwide publications over vaccine safety and efficacy should be encouraged.

\section{CONCLUSION}

The concern for serious side effects was the most common reason of vaccine hesitancy among all age groups including the healthcare workers. The government needs to enhance public trust and share evidence-based knowledge on vaccine efficacy and safety through major sources of COVID-19 information, i.e., television, social media and health care workers. The participants can be convinced to get vaccinated if more published data on vaccine efficacy and safety is available.

\section{LIMITATIONS OF THE STUDY}

Our study findings have certain limitations, as it was online survey hence opinion of digitally illiterate people is underreported. Use of social media for data collection might have affected the variety of the study sample. Nevertheless, as 50\% (159) participants having vaccine hesitancy were doctors, we believe our study findings will be helpful in addressing vaccine hesitancy effectively as health care professionals if convinced can play a pivotal role in fostering acceptance of vaccine among vaccine-hesitant.

\section{CONFLICTS OF INTEREST}

The authors have no competing financial interests or personal relationships to declare.

\section{REFERENCES}

1. Dror, A.A., Eisenbach, N., Taiber, S., Morozov, N.G., Mizrachi, M., Zigron, A., Srouji, S. and Sela, E. (2020) Vaccine Hesitancy: The Next Challenge in the Fight against COVID-19. European Journal of Epidemiology, 35, 775-779. https://doi.org/10.1007/s10654-020-00671-y

2. Vaccine Hesitancy in Pakistan Heightens Risk of COVID Resurgence. https://www.aljazeera.com/news/2021/3/5/in-pakistan-vaccine-hesitancy-heightens-risk-of-covid-19-resurge

3. Khan, Y.H., Mallhi, T.H., Alotaibi, N.H., Alzarea, A.I., Alanazi, A.S., Tanveer, N. and Hashmi, F.K. (2020) Threat of COVID-19 Vaccine Hesitancy in Pakistan: The Need for Measures to Neutralize Misleading Narratives. American Journal of Tropical Medicine and Hygiene, 103, 603-604. https://doi.org/10.4269/ajtmh.20-0654

4. Ali, M., Ahmad, N., Khan, H., Ali, S., Akbar, F. and Hussain, Z. (2019) Polio Vaccination Controversy in Pakistan. The Lancet, 394, 915-916. https://doi.org/10.1016/S0140-6736(19)32101-4

5. Bukhari, M.H., Zain, S. and Akram, J. (2020) Will COVID-19 Vaccine Genetically Modify Humans? Facts and Myths. Biomedica, 36, 329-333. https://doi.org/10.51441/BioMedica/5-72

6. John Hopkins, Health. COVID-19 Vaccine Hesitancy. 
https://www.hopkinsmedicine.org/health/conditions-and-diseases/coronavirus/covid19-vaccine-hesitancy-12-t hings-you-need-to-know

7. Krammer, F., Srivastava, K., Alshammary, H., Amoako, A.A., Awawda, M.H., Beach, K.F., Bermúdez-González, M.C., Bielak, D.A., Carreño, J.M., Chernet, R.L., Eaker, L.Q., Ferreri, E.D., Floda, D.L., Gleason, C.R., Hamburger, J.Z., Jiang, K., Kleiner, G., Jurczyszak, D., Matthews, J.C., Mendez, W.A., Nabeel, I., Mulder, L.C.F., Raskin, A.J., Russo, K.T., Salimbangon, A.T., Saksena, M., Shin, A.S., Singh, G., Sominsky, L.A., Stadlbauer, D., Wajnberg, A. and Simon, V. (2021) Antibody Responses in Seropositive Persons after a Single Dose of SARSCoV-2 mRNA Vaccine. The New England Journal of Medicine, 384, 1372-1374. https://doi.org/10.1056/NEJMc2101667

8. Dogan, M., Kozhaya, L., Placek, L., Gunter, C., Yigit, M., Hardy, R., Plassmeyer, M., Coatney, P., Lillard, K., Bukhari, Z., Kleinberg, M., Hayes, C., Arditi, M., Klapper, E., Merin, N., Liang, B.T., Gupta, R., Alpan, O. and Unutmaz, D. (2021) SARS-CoV-2 Specific Antibody and Neutralization Assays Reveal the Wide Range of the Humoral Immune Response to Virus. Communications Biology, 4, 129. https://doi.org/10.1038/s42003-021-01649-6

9. Jaffe, E., Lyerly, A.D. and Goldfarb, I.T. (2020) Pregnant Women's Perceptions of Risks and Benefits When Considering Participation in Vaccine Trials. Vaccine, 38, 6922-6929. https://doi.org/10.1016/j.vaccine.2020.08.059

10. Wang, K., Long, Q.X., Deng, H.J., Hu, J., Gao, Q.Z., Zhang, G.J., He, C.L., Huang, L.Y., Hu, J.L., Chen, J., Tang, N. and Huang, A.L. (2020) Longitudinal Dynamics of the Neutralizing Antibody Response to SARS-CoV-2 Infection. Clinical Infectious Diseases, ciaa1143.

11. Han, Y., Liu, P., Qiu, Y., Zhou, J., Liu, Y., Hu, X., Yang, Q., Huang, R., Wen, X., Song, H., Yu, P., Yang, M., Zhang, J., Liu, W.J., Peng, K., Wu, G., Zhang, D., Zhou, X. and Wu, Y. (2021) Effective Virus-Neutralizing Activities in Antisera from the First Wave of Survivors of Severe COVID-19. JCI Insight, 6, e146267. https://doi.org/10.1172/jci.insight.146267

12. Fisher, K.A., Bloomstone, S.J. and Walder, J. (2020) Attitudes toward a Potential SARS-CoV-2 Vaccine: A Survey of U.S. Adults. Annals of Internal Medicine, 173, 964-973. https://doi.org/10.7326/M20-3569

13. Aloweidi, A., Bsisu, I., Suleiman, A., Abu-Halaweh, S., Almustafa, M. and Aqel, M. (2021) Hesitancy towards COVID-19 Vaccines: An Analytical Cross-Sectional Study. International Journal of Environmental Research and Public Health, 12, 5111. https://doi.org/10.3390/ijerph18105111

14. Reiter, P.L., Pennell, M.L. and Katz, M.L. (2020) Acceptability of a COVID-19 Vaccine among Adults in the United States: How Many People Would Get Vaccinated? Vaccine, 38, 6500-6507. https://doi.org/10.1016/j.vaccine.2020.08.043

15. Al Kaabi, N., Zhang, Y., Xia, S., et al. (2021) Effect of 2 Inactivated SARS-CoV-2 Vaccines on Symptomatic COVID-19 Infection in Adults: A Randomized Clinical Trial. JAMA. https://doi.org/10.1001/jama.2021.8565

16. Elzein, F., Ibrahim, A., Alshahrani, F., et al. (2021) Reinfection, Recurrence, or Delayed Presentation of COVID19? Case Series and Review of the Literature. Journal of Infection and Public Health. https://doi.org/10.1016/j.jiph.2021.01.002

17. Rizwan, W., Rana, N., Bukhari, M.H. and Sadiq, M. (2021) Vaccine Must Be Provided to Health Professionals Lactating Mothers. Journal of Human Lactation. https://doi.org/10.1177/08903344211021319 